of orogenic folding and intrusion of granite, and it is thought probable that in these early ages, as since, mountain-building has been restricted to narrow belts of the earth's crust.

Absorption Spectra of Complex Salts.-A paper by Dr. R. Samuel in a recent issue of the Zeitschrift für Physik (vol. 70, No. 1) contains much new information on the absorption spectra of complex salts of iron, cobalt, nickel, palladium, and platinum. The measurements have been made both in the visible region and well into the ultra-violet ( $2300 \mathrm{~A}$.), the progress towards shorter wave-lengths being due to an improvement in technique, involving the use of the continuous spectrum of hydrogen as a light source. Graphs are given showing the variation of absorption coefficient with wave-length for twenty-one substances. The chief interest in these experiments is in their possible bearing on the structure of the complex radicles. Dr. Samuel records a number of apparent regularities, such as the existence of three similarly situated bands in the spectra of the diamagnetic cyanides and the appearance of a fourth band at greater wave-length with paramagnetic cyanides. His work, although extensive, is, however, definitely of a preliminary nature. The physics department of the Muslim University of Aligarh, of which Dr. Samuel is the head, is being reorganised and equipped to provide facilities for research in molecular physics, and he is continuing his work there.

Explosions in Closed Cylinders.-There is a pronounced increase in the speed of propagation of flame in a gas mixture in a tube open at both ends when a number of restricting brass rings are arranged within the tube. This effect, observed by Chapman and Wheeler in 1926, has been shown by Kirkby and Wheeler, in the Journal of the Chemical Society for September, to apply also to a closed tube. In explanation of the general effect of restrictions, it has been shown that during the early stages of an explosion in a tube the unburnt mixture ahead of the flame front moves as a current and the speed of the flame depends on the speed of the current. When the restricting rings are close enough together, the tongue of flame issuing from the first ring passes through the second and initiates combustion of the gas beyond while the portion between the two rings is still burning. The combustion of a comparatively large volume of gas is thus almost instantaneous. The effect occurs also in closed tubes, the sudden expansion resulting from the almost simultaneous combustion of the gas in each compartment producing a shock wave.

Rhenium Compounds.-The preparation of rhenium tetrachloride, and the rhenichlorides of potassium and silver, is described by Briscoe, Robinson, and Stoddart in the September number of the Journal of the Chemical Society. Precipitates of insoluble rhenichlorides were also obtained with mercurous and thallous salts. The primary product of treating the metal in chlorine is the tetrachloride, $\mathrm{ReCl}_{4}$, which has not previously been observed. No evidence of the existence of the reported hexa- and hepta-chlorides was obtained, although the black crystalline tetrachloride was always accompanied by traces of a brown, well-crystallised substance, melting sharply at about $21^{\circ}$, obtained in quantities insufficient for analysis. No evidence of the formation of a lower chloride was obtained when rhenium was treated in dry hydrogen chloride up to $900^{\circ}$, the metal being unattacked. Potassium rhenichloride, $\mathrm{K}_{2} \mathrm{ReCl}_{6}$, was obtained in green crystals by heating a mixture of rhenium and potassium chloride in chlorine and crystallising from cold water. The silver salt, $\mathrm{Ag}_{2} \mathrm{ReCl}_{6}$, was obtained as an orange precipitate on adding a slight excess of silver nitrate to an aqueous solution of potassium rhenichloride. It is not obviously crystalline. Preliminary experiments on the behaviour of metallic rhenium when heated with bromine and iodine confirmed the observations of Noddack : the products are regarded as tetrahalides.

\title{
Astronomical Topics.
}

Meteoric Hypothesis for the Origin of the Solar System.-The Abbé Moreux contributes articles on cosmogony to Scientia for September and October. Instead of invoking the approach of another star to the sun, he supposes the sun to have traversed a region of space full of dust or meteoric matter, such as are indicated by the dark 'Horse's Head ' in Orion and a number of similar dark patches. The orbits of such particles about the sun would in general be hyperbolic, but he suggests that by collisions and other mutual interference a considerable part of the matter might be captured by the sun. He traces its subsequent behaviour in some detail, and attempts an explanation of Bode's law of planetary distances. $\mathrm{He}$ notes that the planes of the giant planets are alternately on opposite sides of the invariable plane of the system; he draws an argument from this in support of his system. But the planes are in a state of constant change, and it would seem that proof is required that the relation now existing is permanent before any cosmogonic argument can be drawn from it. In his discussion of Neptune's system he assumes that Neptune's rotation is retrograde, like the motion of the satellite. The observations of J. H. Moore and D. H. Menzel at the Lick Observatory in 1928 showed that the rotation is direct. In such a difficult subject as cosmogony, it is well that all possible suggestions should be examined. It is unlikely that any one of the suggested systems is completely true.
Repetition of the Michelson-Morley Experiment.Six years ago, Prof. D. C. Miller announced the results of a series of experiments that he made on Mt. Wilson; these seemed to indicate a variation of some $9 \mathrm{~km}$./sec. in the course of the year, which he ascribed to a drift of the solar system in a direction nearly normal to the ecliptic. In the "Encyclopædia Britannica" (14th edition, vol. 15, p. 418) are described three series of experiments, by Kennedy and Illingworth, by Piccard and Stahel, and by Michelson, Pease, and Pearson, all made between 1927 and 1929, and all giving a zero effect like the original experiment.

The Scientific American for October gives details of a later series of experiments carried out by Prof. G. Joos, of the University of Jena, using apparatus constructed by the Zeiss works. The path of the light in the apparatus is brought up to 70 feet by repeated reflections. The results are recorded photographically and they are stated to preclude any ether drift exceeding one mile per second, so that it may be assumed to be zero. The large cross of the apparatus is constructed of quartz, for the double reason of its low coefficient of expansion and its freedom from magnetic effects. The cross is suspended by 700 springs of piano-wire, so as to support every part equally and prevent torsion. There is thus a great majority of experiments that indicate a zero effect of ether drift. 